\title{
Aggressive Cardiac Involvement in Systemic Lupus Erythematosus: A Case Report and a Comprehensive Literature Review
}

\author{
Reza Ashrafi, ${ }^{1}$ Pankaj Garg, ${ }^{1}$ Ewan McKay, ${ }^{1}$ John Gosney, ${ }^{2}$ Som Chuah, ${ }^{1}$ and Gershan Davis ${ }^{1}$ \\ ${ }^{1}$ Aintree Cardiac Centre, University Hospital Aintree, Lower Lane, Liverpool L9 7AL, UK \\ ${ }^{2}$ Department of Pathology, The Royal Liverpool University Hospital, Prescot Street, Liverpool L7 8XP, UK
}

Correspondence should be addressed to Reza Ashrafi, reza.ashrafi@aintree.nhs.uk

Received 12 November 2010; Revised 24 December 2010; Accepted 7 January 2011

Academic Editor: Jalal K. Ghali

Copyright (C) 2011 Reza Ashrafi et al. This is an open access article distributed under the Creative Commons Attribution License, which permits unrestricted use, distribution, and reproduction in any medium, provided the original work is properly cited.

\begin{abstract}
Background. We present the case of a 35-year-old gentleman who presented with an aggressive cardiomyopathy with normal coronary arteries. He was later diagnosed with systemic lupus-related cardiomyopathy. Methods. We undertook an extensive review of the literature regarding cardiac manifestations of lupus and used over 100 journals to identify the key points in pathology, diagnosis, and treatment. Results. We have shown that cardiac lupus can be rapidly progressive and, unless treated early, can have severe consequences. The predominant pathologies are immune complex and accelerated atherosclerosis drive. Treatment comprised of high-level immunosuppression.
\end{abstract}

\section{Initial Referral}

A 35-year-old UK born man was referred to the cardiology department at University Hospital Aintree in October 2009 with a few troublesome palpitations a day, which had increased in frequency over the preceding 4 months, and occasional dizziness.

His background was as a law student who was a nonsmoker and consumed no alcohol. His family history revealed a fit and well mother, father, and sister but, interestingly, two paternal uncles who had died suddenly of an unknown cause.

His examination was unremarkable, and his ECG was normal apart from a few isolated unifocal ventricular ectopics and a normal echocardiogram apart from some mild distal inferior septal hypokinesis with an ejection fraction of $57.3 \%$ using the Simpson's biplane method.

Plans were made for him to have an outpatient 24-hour tape and to be reviewed again in clinic.

\section{First Admission}

Unfortunately, he was admitted via the emergency department three days later with constant palpitations, dizziness, and increasing shortness of breath. Clinically, there were no signs of heart failure or thyroid disease.

He was found to have a Troponin T of $1.02 \mu \mathrm{g} / \mathrm{L}$ (1.39 at twelve hours), and his ECG showed sinus rhythm with septal $\mathrm{Q}$ waves, poor septal $\mathrm{R}$ wave progression, and ventricular couplets. While on telemetry, he was noted to have short runs of ventricular tachycardia (VT) with a high background burden of ventricular ectopics.

He was started on treatment for his heart failure and arrhythmia comprising of bisoprolol $2.5 \mathrm{mg}$, aspirin $75 \mathrm{mg}$, and ramipril $1.25 \mathrm{mg}$ (the dosages were maximised as far as his blood pressure would allow).

$\mathrm{He}$ had a repeat echocardiogram, which revealed a worsening of his inferoseptal hypokinesis from mild to severe and some anterior/apicoanterior hypokinesis. He was also noted to have multiple strands/trabeculae in the LV apex.

To further investigate, a 3D echocardiogram was organised which revealed a false tendon but also hypertrabeculation in the apex and a 3-way connection between the inferior, septal, and anterior walls. A diagnosis of LV compaction was mooted, and his angiogram was scheduled for the next day.

A picture of the $3 \mathrm{D}$ echocardiogram, Figure 1 is showing spontaneous echo contrast and trabeculations in the apex 


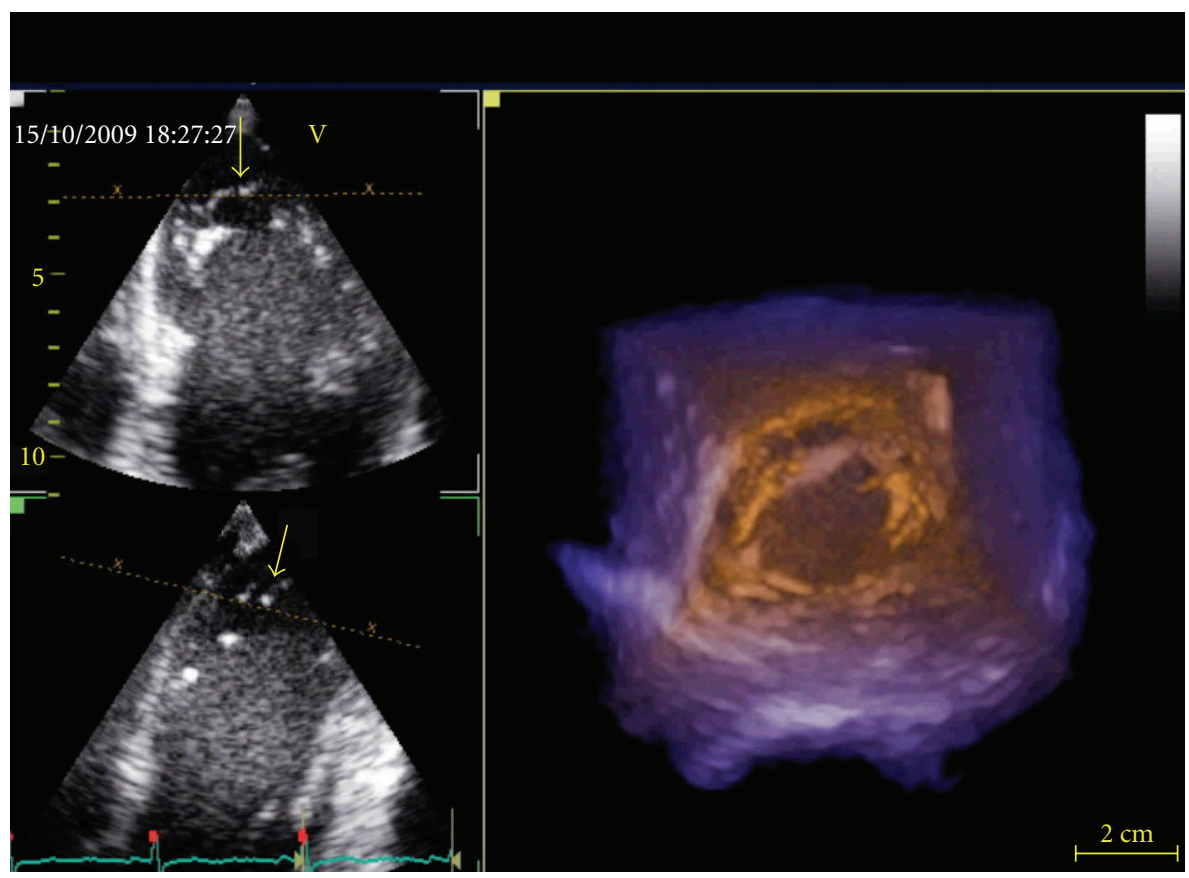

FIGURE 1

A screen for cardiomyopathy revealed normal iron levels, thyroid function, magnesium, zinc, ceruloplasmin, and immunoglobulins. His angiogram revealed unobstructed coronaries with no evidence of thrombosis and no disease that would explain his regional wall motion abnormalities, good compaction on ventriculography, and an ejection fraction of $40 \%$.

Reversible causes of cardiomyopathy were considered and excluded, and there was no history of an infective viral precipitant. The combination of a negative cardiomyopathy screen so far and the lack of an infective cause, made a progressive condition most likely as his symptoms progressed.

He improved symptomatically and was seen two weeks later by the electrophysiology team, who organised an MRI that revealed both left and right ventricular dilatation with moderate biventricular impairment and scarring. An outpatient 24-hour tape revealed a sinus bradycardia with almost 19,000 ventricular ectopics, including couplets and triplets but no ventricular tachycardia.

An image of his cardiac MRI on 2D scar weighting is shown in Figure 2 with scarring and biventricular dilatation.

An implantable cardiac defibrillator was discussed and the patient chose to go away and consider his options. The option of a life vest defibrillator was not considered at that point as it was felt that the patient needed to weigh up the effect of any defibrillator, and if he were to reject an implantable version then the lifevest system would be offered.

His medical treatment remained the same comprising of aspirin, bisoprolol, simvastatin, and ramipril.

\section{Second Admission}

He was readmitted 6 weeks later with increasing frequency of palpitation and debilitating dizziness. During his admission,

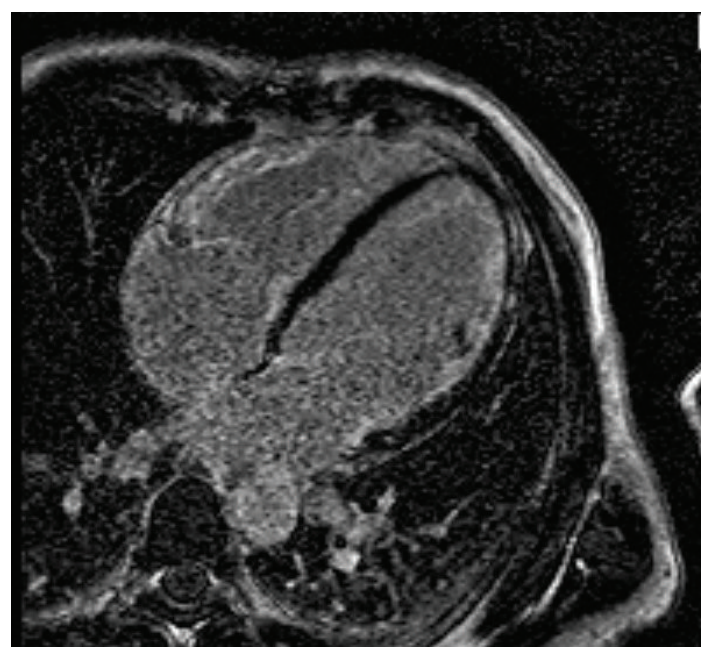

Figure 2

he had frequent runs of VT with occasional haemodynamic compromise before cardioverting on his own.

As the patient's blood pressure was only ever just above $100 \mathrm{~mm} / \mathrm{hg}$, we were unable to increase any of his heart failure treatment.

$\mathrm{He}$ underwent left and right heart catheterisation and endomyocardial biopsies at the local specialist centre before he had an ICD inserted. The left heart catheter was repeated as the team at the specialist centre felt the rapidly progressive nature of his course merited exclusion of coronary artery thrombus formation. The left heart catheter showed normal coronary arteries again, and the right ventricular systolic pressure was 25 and the pulmonary capillary wedge pressure was 10. 


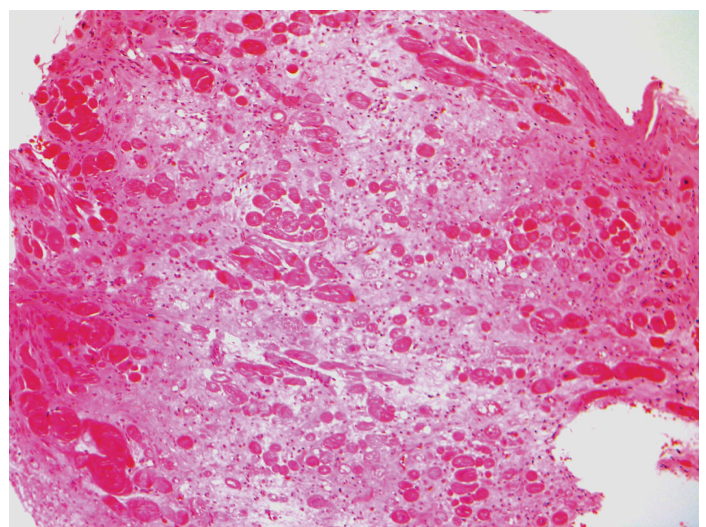

FIGURE 3

He improved in a short period of time with the same medications and was discharged with followup arranged for the results of his biopsy.

\section{Third Admission}

This patient was readmitted two weeks into the year 2010 with chest tightness and orthopnoea, his exercise tolerance dropping to 50 yards. His oxygen saturations on air were $89 \%$, and apart from a displaced apex beat there were no signs of heart failure.

During this time, he was noted to have lost a considerable amount of weight and to have developed a widespread myopathy. He had an autoimmune screen, which was positive for anti-RO and LA antibodies with an ANA titre of $1: 640$ with a membrane speckling pattern and a CK of 10,000 . His lupus coagulant and anticardiolipin antibodies were negative. His forced vital capacity was normal at 5.81. CT pulmonary angiography was negative.

The histology of his cardiac biopsy revealed extensive myocardiolysis and interstitial fibrosis with no abnormal staining for amyloid or iron, a result suggestive of ischaemia consistent with a possible diagnosis of SLE. He went on to have a muscle biopsy, which showed widespread skeletal muscle necrosis. His genetic tests for the various types of muscular dystrophy were negative.

Histology of his cardiac biopsy, showing fibrosis in between the few surviving myocardial fibres, is shown in Figure 3.

He had his echocardiogram repeated, and all his chambers had become dilated with severely impaired LV and RV function with spontaneous echo contrast and an ejection fraction of $16 \%$. His pulmonary artery pressure was 9.4 plus right atrial pressure. This reduction had occurred over a period of 3 months. As a result of severe cardiac impairment and the presence of spontaneous echo contrast, he had his aspirin replaced with warfarin to cover for the high thromboembolic risk.

He continued on the ramipril $1.25 \mathrm{mg}$, bisoprolol $2.5 \mathrm{mg}$ with furosemide $40 \mathrm{mg}$ bd, and spironolactone $25 \mathrm{mg}$ with warfarin added. Unfortunately, despite the maximum antiheart failure treatment his blood pressure would tolerate, he did not make any significant improvement

\section{Most Recent Admission}

He was readmitted two weeks after his most recent discharge again with worsening shortness of breath and fatigue.

After a multidisciplinary discussion with neurology and rheumatology, a diagnosis of systemic lupus erythematosus (SLE) was made, and he was put on high-dose steroids and given a short course of immunoglobulins. He improved clinically with a larger appetite, better mobility, and a reduction in his CK to only 3000 .

He was given 6 cycles of outpatient cyclophosphamide before being put on maintenance Azathioprine $100 \mathrm{~m} /$ day in addition to his existing treatment. Aggressive immunosuppressives helped clinically, and there was a small improvement in his end diastolic diameters and an improvement in his ejection fraction by $2 \%$. As the antiheart-failure treatment remained the same, it was felt that the improvement in his echo parameters was due to his immunosuppression.

\section{Current Condition}

Mr. A has begun to deteriorate once again and is currently being seen by both the transplant and rheumatology teams to see what the benefits and risks of cardiac transplant would be.

\section{Review Methodology}

Three reviewers undertook literature searches using PubMed and MEDLINE with search terms including SLE myocarditis, SLE cardiomyopathy, SLE with cardiac involvement, treatment of SLE cardiomyopathy, and cardiac/heart transplant in SLE. Lupus replaced SLE in all the above searches in a second round to ensure maximum data collection. We excluded animal studies work on patients under 18, and studies which did not focus on the cardiac manifestations of SLE. There were no date restrictions, and only articles in English were used for the review.

Over 100 articles were identified and were then systematically reviewed to extrapolate important applicable points for future use in four key areas: pathology, imaging, treatment, and transplant.

\section{Discussion}

We present below a review of the literature as SLE cardiomyopathy carries a significant mortality risk over SLE without cardiac involvement [1], but it is important to remember that all cardiomyopathy has a poor prognosis with studies looking at cardiomyopathy not related to coronary artery disease having a 5-year mortality of 20\% [2].

We will now look systematically at the incidence, diagnosis, treatment, and outcomes in SLE with cardiac involvement. 
8.1. Incidence and Prevalence. SLE is one of the most common multisystem autoimmune disorders in the world with a variable prevalence but when investigated a prevalence of $51 / 100000$ in 2003 [3]. The male-female ratio is approximately 9:1 with a higher incidence in people of a non-Caucasian background [4].

The incidence of cardiac involvement at postmortem is approximately forty percent, but only six percent of patients had echocardiographic evidence of impairment, and only 1 death in a cohort of over 500 patients was attributable to cardiac involvement [5].

8.2. Diagnosis of SLE: A Noncardiac Perspective. The diagnosis of SLE without the advantage of biopsy specimens remains based on the 1997 update from the American College of Rheumatologists and is based on four positive criteria out of eleven [6] reproduced below:

(1) malar rash,

(2) discoid rash,

(3) photosensitivity,

(4) oral ulcers,

(5) nonerosive arthritis,

(6) pleuritis or pericarditis,

(7) renal disorder,

(8) neurological disorder,

(9) haematological disorder,

(10) immunological disorder, for example, positive antibodies to DS-DNA, SM, or phospholipids,

(11) positive ANA.

As many patients do not make it as far as a definitive biopsy, diagnosis remains based on a combination of clinical features and immunological phenomena as described in the above guidelines.

8.3. Cardiac Pathology in SLE. There are a number of possible cardiac manifestations of SLE, the most common forms being pericarditis, myocarditis, nonbacterial verrucous endocarditis, coronary artery disease, coronary arteritis, premature coronary atherosclerosis, congestive heart failure, cardiac arrhythmias, pulmonary hypertension, and conduction disturbances.

Symptoms and severity of the disease are linked to the pattern of autoantibodies expressed, in addition, the interindividual variable expression of antibodies to ds-DNA, Ro, or anticardiolipin, permit a subtyping of SLE and indicate that there may well be great overlapping of SLE and other conditions such as dermatomyositis, progressive systemic sclerosis, or Sjögren's syndrome.

SLE manifests as we see above in many ways, but we will focus on myocardial disease and accelerated atherosclerosis, as they constitute the major primary cardiac problems.

SLE is the classical example of an immune complex (IC) associated systemic autoimmune disease, and that this immune complex deposition results in an inflammatory response involving the myocardium, pericardium, and perivascular tissue of cardiac blood vessels [7]. This infiltration by immune complexes leads to complement activation and a generalised polymorph invasion followed by myocardial damage [8].

The other major manifestation that we will consider is accelerated atherosclerosis, and it has been with approximately fifty percent of all sufferers having significant atherosclerosis at postmortem [9]. The underlying mechanism is thought to be similar to that of the general population but at an accelerated rate due to inflammatory endothelial dysfunction [8].

The medications involved in treating SLE may also contribute, such as azathioprine-induced endothelial dysfunction [8]. There are also some suggestions as with our patient that there is an alteration of coronary microcirculation leading to ischaemic damage with normal coronaries on angiography similar to our patient.

8.4. Cardiac Imaging in SLE. Before we can begin on imaging, the key first step in investigation of SLE-related cardiac disease is the electrocardiogram for analysis of arrhythmias, ischaemic change, and left ventricular function.

While there is no particular consensus on what imaging is required when cardiac involvement with SLE is suspected, the most reasonable first step is echocardiography as with many cardiac diseases. Echocardiography can help diagnose SLE-related pericarditis, systolic dysfunction, valvular involvement, and cavity thrombus formation with a good sensitivity [10]. While transthoracic echocardiography is more than acceptable in routine practice, sometimes transesophageal echocardiography may be needed for closer analysis of valve structure and function and is thus more sensitive [11]. 3D echocardiography does not really add much to diagnosis except for exclusion of other types of cardiomyopathy such as noncompaction cardiomyopathy.

The next step in investigation, we would suggest, in patients with cardiomyopathy and SLE is coronary angiography as the rate of atherosclerosis is markedly raised compared to control subjects [12]. This is based on the fact that there can be thrombus within the coronary arteries but the main step is to look for significant coronary artery disease causing ischaemia, as this is the most common factor for cardiomyopathy in normal people and myocardial infarction rates are fifty-times higher in SLE [13].

Other imaging modalities have been used to look for ischaemia such as myocardial perfusion scanning [14], cardiac CT angiography [15], and stress echocardiography [16]. As these techniques and cardiac MRI angiography become more widespread in clinical practice, we feel they will to a large extent, replace invasive angiography.

If angiography has shown normal coronary arteries and there is evidence of an SLE cardiomyopathy process, then magnetic resonance imaging should be undertaken to look for changes in structure such as fibrosis and scarring, and in some cases MRI has been used to check function in patients who have clinically improved [17]. 
Endomyocardial biopsy for attempted definitive histological diagnosis despite the risks and potentially low yield is still widely recommended [8], and at least five right ventricular samples have been recommended [18]. It is important to say that biopsy is mainly used to differentiate between SLE myocarditis and antimalarial-induced damage, and in a previously published case series of 58 patients with endomyocardial biopsies in SLE-related cardiomyopathy, $38 \%$ percent were diagnostic of lupus-induced damage, $22 \%$ of drug related damage, and 29\% nonspecific.

Histological features of SLE cardiomyopathy may be nonspecific to some degree, but there are some important typical features to be aware of which include initially inflammatory cell infiltrates, for example CD 68 macrophages, which can be identified using immunohistochemistry [8]. As the disease advances, there is myocardial necrosis, fibrotic replacement, and scarring, all features seen in our patient's histology.

In the end, the variable diagnostic yield nature of endomyocardial combined with the sometimes nonspecific nature of the disease means that diagnosis must be a balanced clinical decision taking into account the history, examination, immunology, imaging, and, if possible, histology.

\subsection{Treatment of Cardiac SLE}

8.5.1. Medical Management. While SLE causes a wide range of cardiovascular diseases, the complication should be dealt with in the standard fashion, that is, angiotensin-converting enzyme inhibitors, beta-blocker for left ventricular impairment, and coronary stenting for stenoses.

Reviewing the literature, the most common immunosuppressive treatment choice for acute flares seems to be high-dose steroids followed by pulsed cyclophosphamide $[19,20]$, which is a similar regimen to other acute SLE crises such as lupus nephritis. Intravenous immunoglobulins [21] have been used in one or two case reports with some success, but the overwhelming treatment goal should be rapid immunosuppression whatever the method.

After treatment of the acute phase, there seems to be no real consensus on what is the appropriate long-term drug choice, with steroids and azathioprine among the choices [22].

When there is secondary antiphospholipid syndrome, a recent consensus statement suggests warfarin in the case of symptomatic valvulopathy, thrombus, and pulmonary hypertension only and not as a routine [23].

8.5.2. Resynchronisation Therapy. One potential therapeutic option in advanced stages of heart failure regardless of the source is cardiac resynchronisation therapy (CRT). There have been several reports illustrating the successful use of cardiac resynchronization in patients with SLE and resistant cardiomyopathy $[24,25]$. As yet there is much discussion about which patients benefit from resynchronisation, but while there is not complete agreement about patient selection, most groups agree that those with ventricular dyssynchrony, reduced ejection fraction, and symptomatic
New York classification 2 to 3 heart failure benefit most [26]. One interesting potential unexpected benefit of CRT is that it has been shown to reduce inflammatory cytokines, possibly reducing the inflammatory process [27].

Overall SLE myocarditis has a much poorer outcome when compared to primary myocarditis [28], and this illustrates the need for prompt diagnosis and early aggressive treatment, which can result in improvement of myocardial function back to normal [19].

8.6. Cardiac Transplant in SLE. Our search results revealed a total of 7 cases of cardiac transplant in SLE for a variety of reasons, 4 were for pulmonary hypertension, 1 for postmitral valve replacement heart failure, 1 for cardiac failure following prolonged infective endocarditis in a patient with SLE, and 1 patient with myocarditis and heart failure [29, 30]. Transplant was successful in 5 of the seven cases with followup through both cohorts being at least 14 months $[29,30]$, and in the case of the patient with myocarditis 18 months with no signs of recurrence on endomyocardial biopsy and EF of 50-60\% [30].

While these are small numbers, they do, at least, give some guidance to physicians that in refractory cases transplant is an option and that there have been positive results in the small number of patients that have been operated on. The ideal postoperative combination of antirejection treatment is normally decided by the local transplant team involved, but in the series, that we reviewed that cyclosporin, prednisolone, and azathioprine in combination was the most widely used regimen $[30,31]$.

\section{Conclusions}

Cardiac SLE is a complex disease with multiple possible pathological processes that requires prompt investigation and prompt treatment, otherwise the damage is unrecoverable as proven in our patient.

\section{References}

[1] M. Apte, G. McGwin Jr., L. M. Vilá, R. A. Kaslow, G. S. Alarcón, and J. D. Reveille, "Associated factors and impact of myocarditis in patients with SLE from LUMINA, a multiethnic US cohort," Rheumatology, vol. 47, no. 3, pp. 362-367, 2008.

[2] C. Cevik, K. Nugent, A. Perez-Verdia, and R. D. Fish, "Prophylactic implantation of cardioverter defibrillators in idiopathic nonischemic cardiomyopathy for the primary prevention of death: a narrative review," Clinical Cardiology, vol. 33, no. 5, pp. 254-260, 2010.

[3] S. Bernatsky, L. Joseph, C. A. Pineau, R. Tamblyn, D. E. Feldman, and A. E. Clarke, "A population-based assessment of systemic lupus erythematosus incidence and prevalenceresults and implications of using administrative data for epidemiological studies," Rheumatology, vol. 46, no. 12, pp. 1814-1818, 2007.

[4] A. Rasmussen, S. Sevier, J. A. Kelly et al., "The lupus family registry and repository," Rheumatology, vol. 50, no. 1, pp. 47$59,2011$.

[5] S. Busteed, P. Sparrow, C. Molloy, and M. G. Molloy, "Myocarditis as a prognostic indicator in systemic lupus 
erythematosus," Postgraduate Medical Journal, vol. 80, no. 944, pp. 366-367, 2004.

[6] American College of Rheumatology, "1997 Update of the 1982 American College of Rheumatology Revised Criteria for Classification of Systemic Lupus Erythematosus," http://www.rheumatology.org/practice/clinical/classification/ SLE/1997_update_of_the_1982_acr_revised_criteria_for_ classification_of_sle.pdf.

[7] A. Tincani, C. B. Rebaioli, M. Taglietti, and Y. Shoenfeld, "Heart involvement in systemic lupus erythematosus, antiphospholipid syndrome and neonatal lupus," Rheumatology, vol. 45, supplement 4, pp. iv8-iv13, 2006.

[8] D. Jain and M. K. Halushka, "Cardiac pathology of systemic lupus erythematosus," Journal of Clinical Pathology, vol. 62, no. 7, pp. 584-592, 2009.

[9] M. R. Hejtmancik, J. C. Wright, R. Quint, and F. L. Jennings, "The cardiovascular manifestations of systemic lupus erythematosus," American Heart Journal, vol. 68, no. 1, pp. 119-130, 1964.

[10] A. Doria, L. Iaccarino, P. Sarzi-Puttini, F. Atzeni, M. Turriel, and M. Petri, "Cardiac involvement in systemic lupus erythematosus," Lupus, vol. 14, no. 9, pp. 683-686, 2005.

[11] M. Hojnik, J. George, L. Ziporen, and Y. Shoenfeld, "Heart valve involvement (Libman-Sacks endocarditis) in the antiphospholipid syndrome," Circulation, vol. 93, no. 8, pp. 1579-1587, 1996.

[12] J. E. Salmon and M. J. Roman, "Accelerated atherosclerosis in systemic lupus erythematosus: implications for patient management," Current Opinion in Rheumatology, vol. 13, no. 5, pp. 341-344, 2001.

[13] S. Manzi, E. N. Meilahn, J. E. Rairie et al., "Age-specific incidence rates of myocardial infarction and angina in women with systemic lupus erythematosus: comparison with the Framingham study," American Journal of Epidemiology, vol. 145 , no. 5, pp. 408-415, 1997.

[14] M. Nikpour, M. B. Urowitz, D. Ibanez, and D. D. Gladman, "Relationship between cardiac symptoms, myocardial perfusion defects and coronary angiography findings in systemic lupus erythematosus," Lupus. In press.

[15] Y. H. Rho, C. P. Chung, A. Oeser et al., "Novel cardiovascular risk factors in premature coronary atherosclerosis associated with systemic lupus erythematosus," Journal of Rheumatology, vol. 35, no. 9, pp. 1789-1794, 2008.

[16] S. Codish, N. Liel-Cohen, M. Rovner, S. Sukenik, and M. AbuShakra, "Dobutamine stress echocardiography in women with systemic lupus erythematosus: increased occurrence of left ventricular outflow gradient," Lupus, vol. 13, no. 2, pp. 101104, 2004.

[17] J. A. Singh, P. K. Woodard, V. G. Dávila-Román et al., “Cardiac magnetic resonance imaging abnormalities in systemic lupus erythematosus: a preliminary report," Lupus, vol. 14, no. 2, pp. 137-144, 2005.

[18] M. J. Fairfax, T. G. Osborn, G. A. Williams, C. C. Tsai, and T. L. Moore, "Endomyocardial biopsy in patients with systemic lupus erythematosus," Journal of Rheumatology, vol. 15, no. 4, pp. 593-596, 1988.

[19] W. G. Law, B. Y. Thong, T. Y. Lian, K. O. Kong, and H. H. Chng, "Acute lupus myocarditis: clinical features and outcome of an oriental case series," Lupus, vol. 14, no. 10, pp. 827-831, 2005.

[20] J. W. Chung, D. Y. Joe, H. J. Park, H. A. Kim, H. S. Park, and C. H. Suh, "Clinical characteristics of lupus myocarditis in Korea," Rheumatology International, vol. 28, no. 3, pp. 275280, 2008 .
[21] Y. Sherer, Y. Levy, and Y. Shoenfeld, "Marked improvement of severe cardiac dysfunction after one course of intravenous immunoglobulin in a patient with systemic lupus erythematosus," Clinical Rheumatology, vol. 18, no. 3, pp. 238-240, 1999.

[22] I. H. Tarner, U. Lange, K. Madlener et al., "A wolf in sheep's clothing: atypical systemic lupus erythematosus (SLE) presenting as cardiovascular disease," Medizinische Klinik, vol. 105, no. 4, pp. 300-304, 2010.

[23] M. Lockshin, F. Tenedios, M. Petri et al., "Cardiac disease in the antiphospholipid syndrome: recommendations for treatment. Committee consensus report," Lupus, vol. 12, no. 7, pp. 518-523, 2003.

[24] P. M. Seferović, A. D. Ristić, R. Maksimović et al., "Cardiac arrhythmias and conduction disturbances in autoimmune rheumatic diseases," Rheumatology, vol. 45, supplement 4, pp. iv39-iv42, 2006.

[25] M. Peregud-Pogorzelska, J. Kaźmierczak, and Z. KornacewiczJach, "Heart failure in systemic lupus erythematosus treated by cardiac resynchronization: a case report," Angiology, vol. 58, no. 2, pp. 238-241, 2007.

[26] P. E. Vardas, A. Auricchio, J. J. Blanc et al., "Guidelines for cardiac pacing and cardiac resynchronization therapy: the Task Force for Cardiac Pacing and Cardiac Resynchronization Therapy of the European Society of Cardiology. Developed in collaboration with the European Heart Rhythm Association," European Heart Journal, vol. 28, no. 18, pp. 2256-2295, 2007.

[27] G. N. Theodorakis, P. Flevari, C. Kroupis et al., "Antiinflammatory effects of cardiac resynchronization therapy in patients with chronic heart failure," Pacing and Clinical Electrophysiology, vol. 29, no. 3, pp. 255-261, 2006.

[28] T. C. Pulerwitz, T. P. Cappola, G. M. Felker, J. M. Hare, K. L. Baughman, and E. K. Kasper, "Mortality in primary and secondary myocarditis," American Heart Journal, vol. 147, no. 4, pp. 746-750, 2004.

[29] A. Colli, C. A. Mestres, G. Espinosa et al., "Heart valve surgery in patients with the antiphospholipid syndrome: analysis of a series of nine cases," European Journal of Cardio-thoracic Surgery, vol. 37, no. 1, pp. 154-158, 2010.

[30] N. Tweezer-Zaks, G. Zandman-Goddard, M. Lidar, Y. HarZahav, A. Livneh, and P. Langevitz, "A long-term follow-up after cardiac transplantation in a lupus patient: case report and review of the literature," Annals of the New York Academy of Sciences, vol. 1110, pp. 539-543, 2007.

[31] R. D. Levy, A. J. Guerraty, M. H. Yacoub, and R. Loertscher, "Prolonged survival after heart-lung transplantation in systemic lupus erythematosus," Chest, vol. 104, no. 6, pp. 19031905, 1993. 


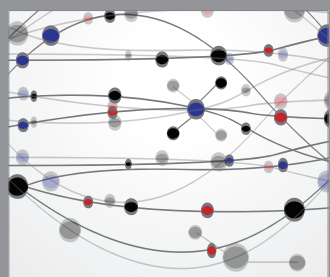

The Scientific World Journal
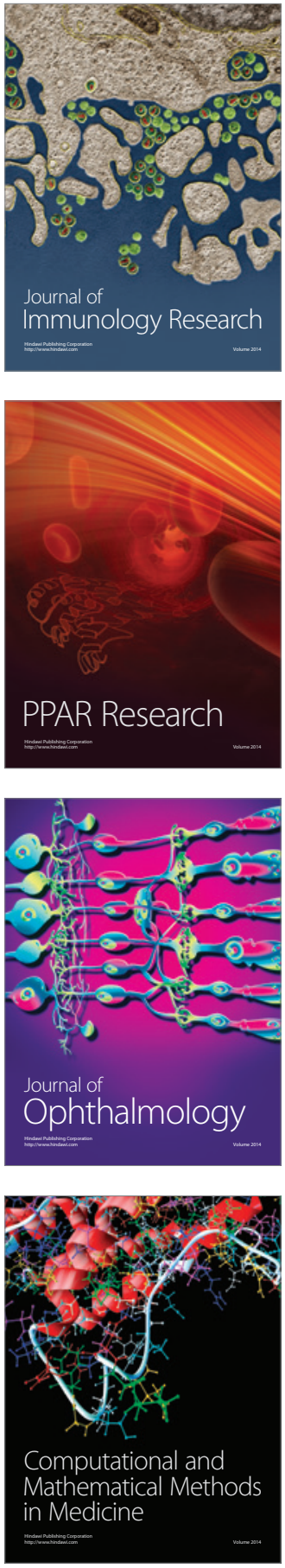

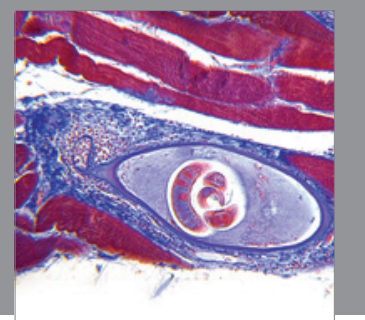

Gastroenterology

Research and Practice
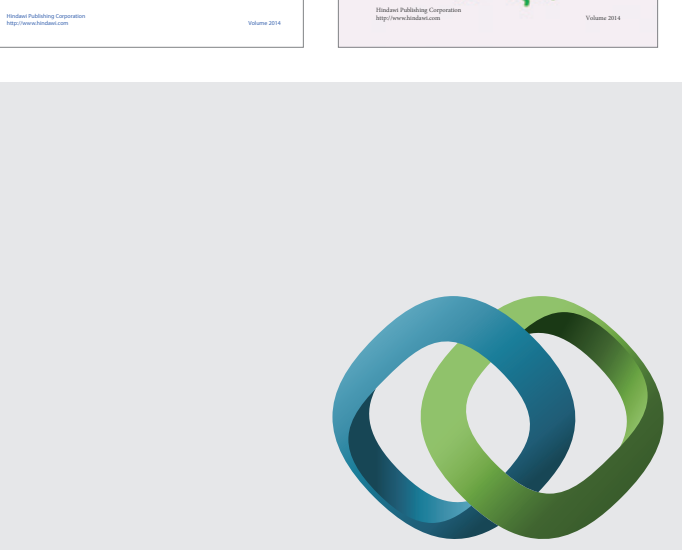

\section{Hindawi}

Submit your manuscripts at

http://www.hindawi.com
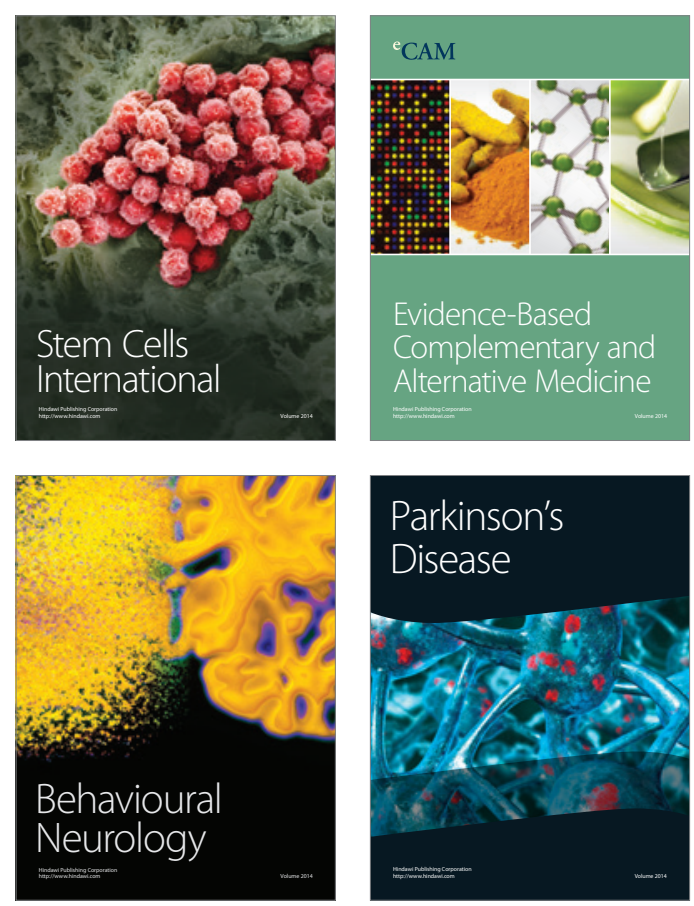

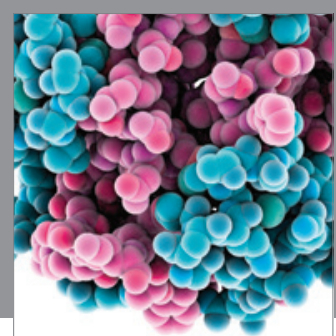

Journal of
Diabetes Research

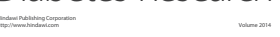

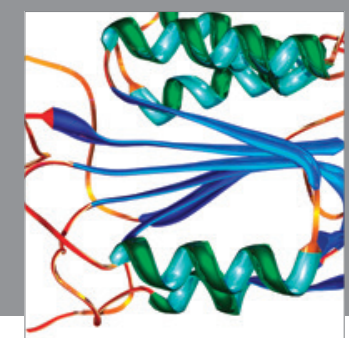

Disease Markers
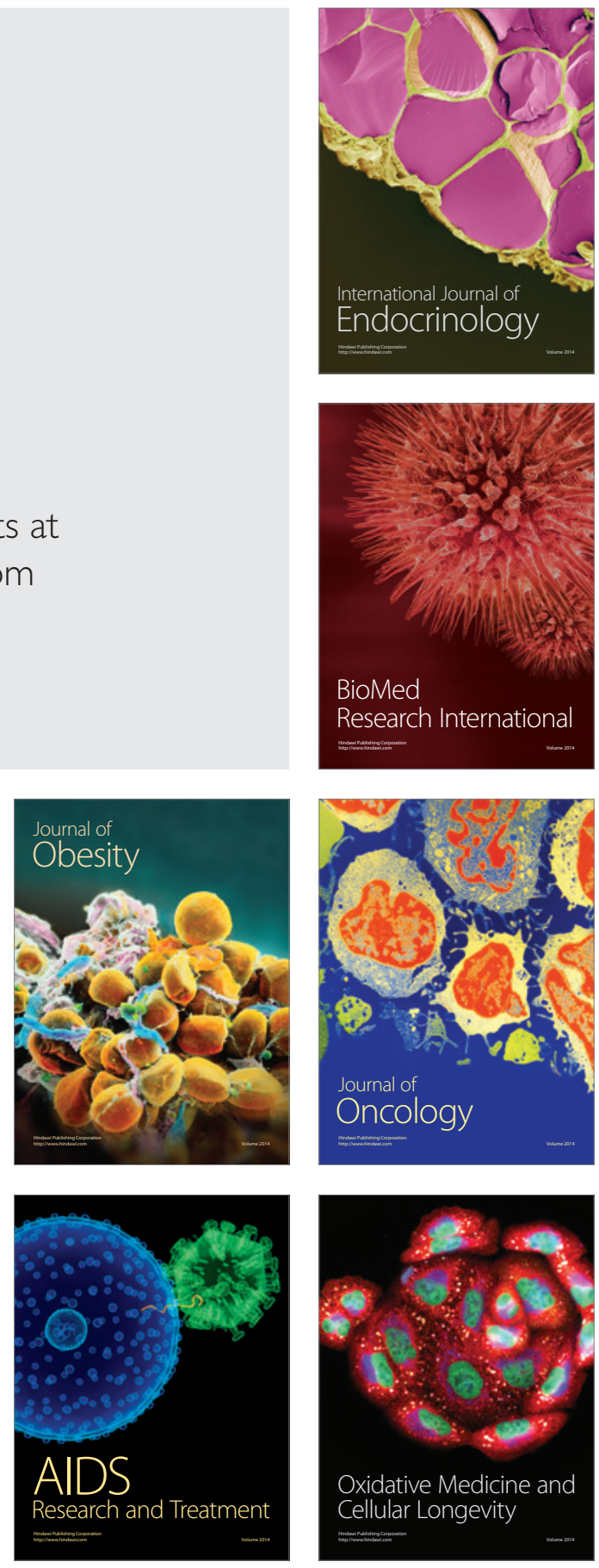